\title{
Morphological assessment of preimplantation embryo quality in cattle
}

\author{
P. Maddox-Hyttel ${ }^{1}$, J. O. Gjørret ${ }^{1}$, G. Vajta ${ }^{2}$, N. I. Alexopoulos ${ }^{3}$, \\ I. Lewis ${ }^{3}$, A. Trounson ${ }^{3}$, D. Viuff ${ }^{4}, 5$, J. Laurincik ${ }^{6}$, M. Müller ${ }^{7}$, \\ P. Tveden-Nyborg ${ }^{\top}$ and P. D. Thomsen ${ }^{1}$
}

'Department of Anatomy and Physiology, The Royal Veterinary and Agricultural University, Groennegaardsvej 7, DK-1870 Frederiksberg C, Denmark; ${ }^{2}$ Section of Reproductive Biology, Department of Animal Breeding and Genetics, Danish Institute of Agricultural Sciences, Research Centre Foulum, DK-8830 Tjele, Denmark; ${ }^{3}$ Monash Institute of Reproduction and Development,

Monash Medical Centre, 246 Clayton Road, Clayton, Victoria 3168, Australia; ${ }^{4}$ Molecular Pharmacology, NovoNordisk A/S, NovoNordisk Park, 2760 Măløv, Denmark: ${ }^{5}$ Department for Clinical Studies, Reproduction, The Royal Veterinary and Agricultural University, Ridebanevej 12.

DK-1870 Frederiksberg C, Denmark; ${ }^{6}$ Constantin the Philosopher University and Research Institute of Animal Production, SK-949 92 Nitra, Slovak Republic; and Veterinary University Vienna, Vienna 1210, Austria

The extensive use of embryo technologies has emphasized the need for assessing embryo quality by morphological techniques, such as transmission electron microscopy, immunocytochemistry for confocal laser scanning microscopy and fluorescence in situ hybridization. By a combination of these techniques, it has been possible to demonstrate: (i) that rRNA gene activation, as monitored by embryonic nucleolar development, is comparable in bovine embryos developed in vivo and produced in vitro, whereas reconstructed nuclear transfer embryos may be deviant, (ii) that generating embryos by both in vitro production and reconstruction by nuclear transfer is associated with increased occurrence of apoptosis, in particular in the inner cell mass of blastocysts, and (iii) that these two embryo production techniques are associated with increased occurrence of mixoploidy that is, embryos presenting a large population of normal diploid cells and a small population of abnormal haploid or polyploid cells. It is clear that blastocysts that appear healthy at stereomicroscopy may have subcellular defects. Therefore, the possibility of long-term evaluation in vitro of embryos after hatching has been examined. However, whereas embryos developing in vivo after hatching present a number of well defined developmental milestones, such as elongation of the trophoblast, formation of hypoblast and epiblast followed by differentiation of endoderm, mesoderm and ectoderm, in vitro culture systems for development beyond the blastocyst stage currently allow the embryo to complete only a single milestone, namely hypoblast formation. 


\section{Introduction}

With the increased use of embryo technology in both breeding and research, assessment of embryo quality has become a key issue. It is clear that techniques such as embryo production in vitro and, in particular, reconstruction by nuclear transfer affect the developmental competence, often referred to as the quality, of embryos (McEvoy et al., 2001). In the long term, development of non-invasive techniques for determining which embryos to transfer and which to discard is an essential goal. However, on a more immediate basis, the use of invasive techniques for assessing, understanding and eliminating the pitfalls inherent to different embryo production techniques is crucial.

Over the past decade, progress has been made with the use of a combination of morphological and molecular techniques for evaluating embryo quality. The morphological approach has been expanded by application of different methods, for example for localization of proteins and nucleic acids by immunocytochemistry and fluorescence in situ hybridization (FISH), respectively. Moreover, the optical possibilities of imaging specimens have also improved with the introduction of techniques, such as confocal laser scanning microscopy (CLSM), allowing for optical sectioning of embryos in toto. Together, these advances have given rise to the area defined as bioimaging.

In this review, the use of different aspects of bioimaging for the assessment of embryo quality are presented with focus on the use of: (i) transmission electron microscopy (TEM), CLSM immunocytochemistry and FISH for studying the activation of the embryonic genome as evaluated through nucleolar development; (ii) TdT-mediated dUTP nick-end labelling (TUNEL) (detection of DNA degradation) and CLSM assessing the occurrence of apoptosis; (iii) FISH for determining chromosome abnormalities; and (iv) TEM and light microscopy for evaluating embryonic development after hatching.

\section{Nucleolar development}

The initial development of mammalian preimplantation embryos is governed by gene transcripts and polypeptides produced by, and stored in, the oocyte during its development (for a review, see Schultz, 1993). However, gradually, the embryonic genome formed at fertilization is activated and assumes control. In cattle, minor activation of the genome is observed already during the first and second cell cycle after fertilization, that is, the one- to twocell embryo (Plante et al., 1994; Hyttel et al., 1996; Viuff et al., 1996; Memili and First, 2000), and this low level of transcription is followed by a major burst during the fourth cell cycle, that is, the eight-cell embryo (Camous et al., 1986; King et al., 1988; Kopecny et al., 1989).

Adequate numbers of ribosomes are required to translate mRNAs inherited from the oocyte as well as those produced de novo from the embryonic genome. Ribosomes are synthesized in the oocyte up to the end of the growth phase at a follicular diameter of about $3 \mathrm{~mm}$ (Fair et al., 1995, 1996). Thus, an initial pool of ribosomes is inherited from the oocyte. The synthesis of ribosomes includes transcription of the ribosomal RNA (rRNA) genes, processing of the transcripts and assembly of the product with proteins to the ribosomal subunits. All of these processes occur in the nucleolus, which is the most prominent entity of the nucleus and, therefore, it may serve as a morphological marker for the activation of the embryonic genome (Kopecny and Niemann, 1993). The functional nucleolus contains three main ultrastructural components (for a review, see Wachtler and Stahl, 1993); the two fibrillar components, consisting of the fibrillar centres and the dense fibrillar component, and the granular component. These components of the so-called fibrillo-granular 
nucleolus reflect the steps in the biosynthesis of ribosomes according to the following model: the fibrillar centres house the enzymatic apparatus for transcription; the dense fibrillar component carries the nascent unprocessed transcripts; and the granular component represents processed transcripts associated with proteins in the form of pre-ribosomal particles. According to this model, the transcription of the rRNA genes is believed to occur at the interface between the fibrillar centres and the dense fibrillar component (Hozak et al., 1994).

The onset of transcription of the rRNA genes in bovine embryos produced in vitro was visualized by using. FISH with a probe hybridizing to the rRNA genes and their transcripts (Viuff et al., 1998). The de novo synthesized rRNA was detected at the sites of the rRNA genes during the third cell cycle that is, the four-cell embryo stage. As revealed by TEM in a parallel study, this initial activation was not accompanied by formation of functional nucleoli and, thus, by ribosome synthesis (Laurincik et al., 2000). However, during the fourth cell cycle, nucleoli were formed signalling the onset of embryonic ribosome synthesis. The nucleoli developed in so-called nucleolus precursor bodies that are already present in the embryo from the first cell cycle (Fig. 1). Initially, tiny fibrillar centres surrounded by the dense fibrillar component are formed peripherally in the precursors, and later during the cell cycle, the granular component develops in the substance of the precursor resulting in a functional fibrillo-granular nucleolus (Laurincik et al., 2000). The chronology and morphology of nucleolar formation is apparently similar in bovine embryos produced in vitro (Laurincik et al., 2000) and developed in vivo (Hyttel et al., 2001a). Whether there is the same similarity with respect to the activation of the rRNA genes during the third cell cycle has not been investigated. In contrast, it has been reported that bovine embryos reconstructed by nuclear transfer from embryonic cells displayed a delay in nucleolar formation to the fifth cell cycle (Hyttel et al. 2001b), whereas those reconstructed from granulosa cells displayed premature nucleolar formation during the third cell cycle (Laurincik et al, 2002). In contrast, Baran et al. (2002) reported a delay of development of fibrillo-granular nucleoli until the fifth cell cycle in bovine embryos reconstructed from fetal fibroblasts as compared with bovine embryos reconstructed from embryonic cells that exhibited functional nucleoli during the fourth cell cycle.

From the molecular perspective, the nucleolus consists of the rRNA genes and their transcripts associated with proteins that play different roles in rRNA transcription, processing and ribosome assembly. Recently, the allocation of key proteins involved in rRNA transcription (topoisomerase I, RNA polymerase 1 and upstream binding factor (UBF)), initial rRNA processing (fibrillarin) or later rRNA processing or nucleolar transport (nucleolin (C23) and nucleophosmin (B23)) to the developing nucleolus in bovine embryos was investigated by immunocytochemistry and CLSM (Laurincik et al., 2000; Hyttel et al., 2001a). During the first three cell cycles, some of the nucleolar proteins were localized to small nuclear entities as described by Baran et al. (1996). However, during the fourth cell cycle, topoisomerase I, RNA polymerase I, UBF and fibrillarin became localized to clusters of presumptive fibrillar centres and fibrillar component, and nucleolin and nucleophosmin to the periphery of the developing nucleoli in correspondence with the TEM observations referred to above (Fig, 2). The chronology and morphology of protein allocation was apparently similar in bovine embryos produced in vitro (Laurincik et al., 2000) and embryos developed in vivo (Hyttel et al., 2001a). However, about half of the embryos produced by nuclear transfer from embryonic (Hyttel et al., 2001b) and granulosa cells (Laurincik et al., 2002) lacked allocation of, in particular, UBF to the developing nucleoli even during the fifth cell cycle, that is the tentative 16-cell embryo. Later stages have not been investigated. 
(a)
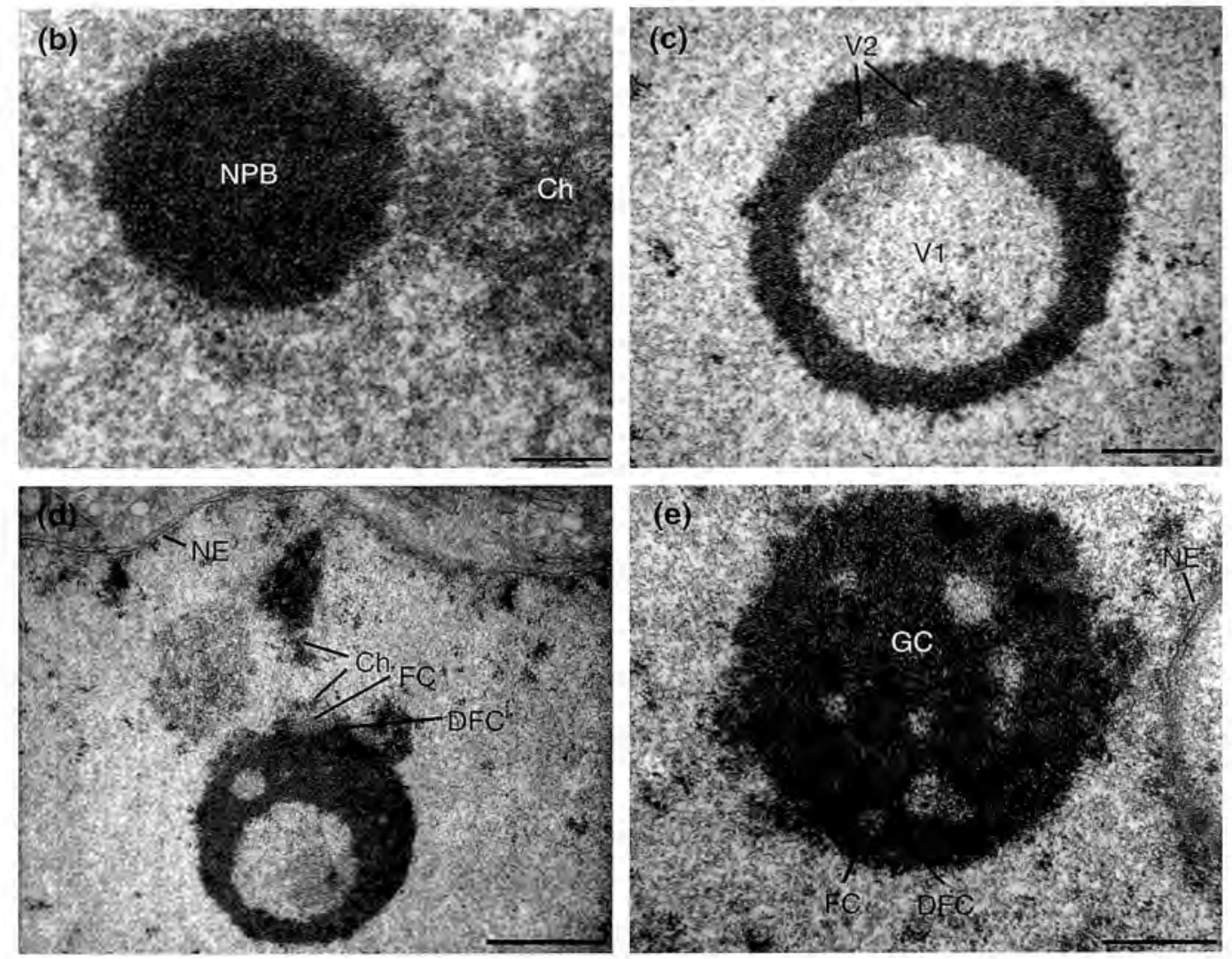

Fig. 1. Light micrograph of (a) a bovine zygote fertilized in vivo (first cell cycle) presenting two pronuclei, one of which displays a nucleolus precursor body (arrow). Transmission electron micrographs of nucleolar development in bovine embryos developed in vivo during the (b) first, (c) third, (d) early fourth and (e) fifth cell cycle. (b) During the first cell cycle, the spherical nucleolus precursor body (NPB) is associated with chromatin (Ch). (c) During the third cell cycle, the NPB displays a primary eccentrical vacuole (V1) and smaller peripheral secondary vacuoles (V2). (d) During the fourth cell cycle, a fibrillar centre (FC), which is associated with chromatin (Ch) and surrounded by dense fibrillar component (DFC), is seen close to the nuclear envelope (NE). (e) During the fifth cell cycle, a spherical fibrillo-granular nucleolus has developed adjacent to the nuclear envelope (NE) presenting fibrillar centres (FC), dense fibrillar component (DFC) and granular component (GC). Scale bars represent (a) $25 \mu \mathrm{m}$, (b) $0.2 \mu \mathrm{m}$, (c) $0.5 \mu \mathrm{m}$, (d) $1.0 \mu \mathrm{m}$ and (e) $0.5 \mu \mathrm{m}$. 
Cell cycle:

2
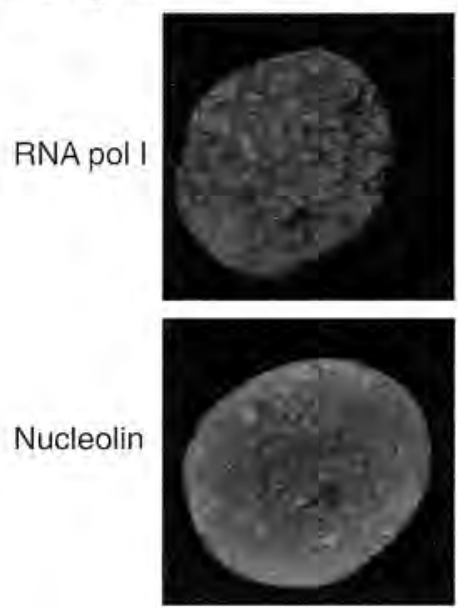

3
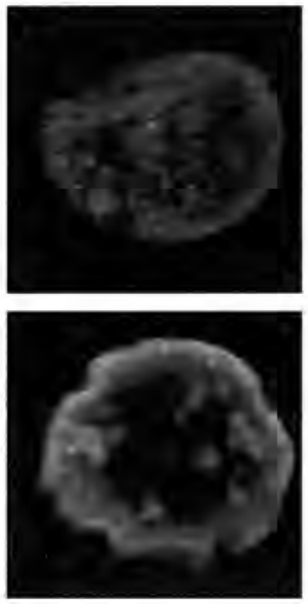

4
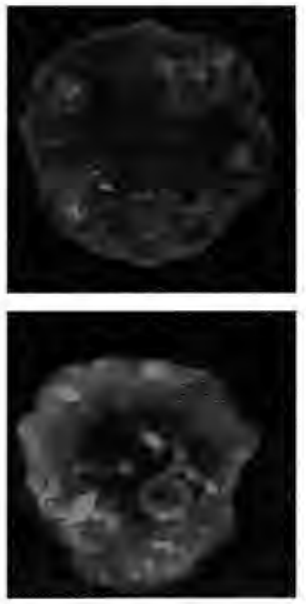

5
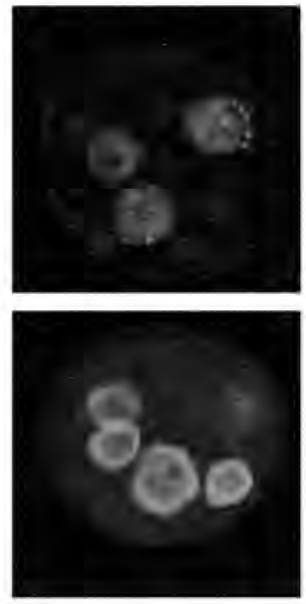

Fig. 2. Confocal laser scanning images of single nuclei from bovine embryos developed in vivo during the second, third, fourth and fifth cell cycle. In all images, the DNA has been stained with propidium iodide (red), whereas in the upper panel RNA polymerase I has been immunolocalized with fluorescein isothiocyanate (FITC) (green) and in the lower panel nucleolin has been immunolocalized with FITC (green). Note that RNA polymerase I localize to clusters of foci (presumptive fibrillar centres) during the fourth and, in particular, the fifth cell cycle and that nucleolin during the same cycles localize to the periphery of the developing nucleoli.

\section{Apoptosis}

Apoptosis, a regulated form of cell death, has been observed in preimplantation embryos from many mammalian species (for a review, see Hardy, 1997, 1999). This phenomenon has received increasing attention for its potential role in early embryonic loss. In embryos after compaction, there is an inverse relationship between the incidence of cell death and the number of cells in murine (Brison and Schultz, 1997; Devreker and Hardy, 1997), bovine (Byrne et al., 1999) and human embryos (Hardy et al., 1989). Furthermore, the incidence of cell death is higher in blastocysts produced in vitro than in blastocysts that are derived in vivo in mice (Brison and Schultz, 1997), and it is well documented that culture conditions have a substantial impact on both the number of cells and the cell death in bovine (Byrne et al., 1999; Makarevich and Markkula, 2002), mouse (Brison and Schultz, 1997; Devreker and Hardy, 1997) and rat embryos (Pampfer et al., 1997). Supplementation of culture media with substances that increase the number of cells in the embryo and rates of blastocyst development, such as insulin-like growth factor I (IGF-I), resulted in a decrease incidence of cell death (Makarevich and Markkula, 2002), and the opposite effect can be observed with for example glucose (Moley et al., 1998). Adverse conditions in vivo have also been documented to both increase cell death and decrease implantation rates in diabetic rodent models (Pampfer et al., 1997; Moley et al., 1998). Thus, the incidence of cell death can be modulated by environmental conditions both in vivo and in vitro.

Generally, the two classical types of cell death, namely necrosis and apoptosis, can be distinguished by differences in morphology (Wyllie et al., 1980). However, it is becoming evident that there are several intermediate forms of cell death (Leist and Jaattela, 2001). Necrosis is an accidental form of cell death and is regarded as a cellular response to pathological insults, whereas apoptosis is a much more regulated and suicidal form, which may represent a physiological and controlled pathway for cellular demise. 
The typical changes in nuclear morphology associated with apoptosis include chromatin condensation and marginalization as well as nuclear fragmentation by karyorhexis (Wyllie et al., 1980). These changes have been extensively used as markers of cellular death in embryos (for review, see Hardy, 1997). Many molecular elements of the apoptotic process have been identified including activation of a protease family known as caspases as well as extensive internucleosomal DNA fragmentation (Hengartner, 2000). Development of techniques for in situ detection of DNA degradation (TUNEL; Gavrieli et al., 1992) has made this parameter available as a molecular marker for assessment of cell death, and it has been used in preimplantation embryos in several species (Jurisicova et al., 1996; Brison and Schultz, 1997; Long et al., 1998; Byrne et al., 1999). However, TUNEL labelling has also been observed in cells undergoing necrotic cell death (Grasl-Kraupp et al., 1995), and it is now evident that it is necessary to use additional markers of apoptosis, for example nuclear morphology, in combination with TUNEL labelling to confirm the apoptotic nature of cell death.

In a recent study, a combination of nuclear morphology and TUNEL labelling was used to estimate the incidence and localization of apoptotic cell death to the trophoblast and inner cell mass (ICM) in bovine blastocysts produced either in vitro in a co-culture system and collected on day 7 after insemination, or flushed from superovulated cows (in vivo) at day 7 after ovulation (Fig. 3). In this study, 11 blastocysts from each production system were analysed (Gjorret et al., 2001). No difference in the total number of cells could be observed between blastocysts derived in vivo and blastocysts produced in vitro, but the in vivo ICMs contained significantly more cells (102.0 \pm 15.2$)$ than in vitro ICMs (70.4 \pm 6.2$)$. All embryos contained apoptotic cells. An apoptotic index was calculated as the number of apoptotic nuclei $\times 100$, divided by the total number of nuclei. This index was significantly higher in blastocysts produced in vitro $(10.1 \pm 1.4)$ than in blastocyts developed in vivo $(6.5 \pm 0.8)$, and this difference was based on a marked significant difference in the apoptotic indices of the ICM (in vitro: $19.3 \pm 3.6$ versus in vivo: $7.1 \pm 1.2$ ) rather than in the trophoblast $(4.5 \pm 1.1$ versus $4.4 \pm 1.4$; non-significant; Gjørret et al., 2001).

In a another study, the chronological appearance of the two apoptotic markers that is, nuclear morphology and TUNEL labelling, in two-cell, three-to eight-cell, 9-16-cell, morula and blastocyst stage bovine embryos with normal developmental kinetics either reconstructed by nuclear transfer from serum starved granulosa cells or derived in vivo from superovulated cows were investigated (Fig. 3). The first apoptotic nuclei were observed in a six-cell nuclear transfer embryo, and they were seen in all subsequent developmental stages (Gjørret et al., 2002). In contrast, apoptotic nuclei could not be observed until the morula stage in embryos derived in vivo. Nuclei displaying only morphological changes, that is, condensation or fragmentation, could be observed in nuclear transfer embryos at all stages investigated, but not before the eight-cell stage in embryos derived in vivo. At the morula stage, the nuclear transfer embryos had an apoptotic index of $8.5 \pm 2.5$, which was significantly higher than the $1.7 \pm 0.5$ of morulae derived in vivo (J. O. Gjørret, $H_{+} \mathrm{M}$. Knijn, S. J. Dieleman, B. Avery, L-I. Larsson and P. Maddox-Hyttel, unpublished). All blastocysts investigated contained at least one apoptotic nucleus, but again the nuclear transfer embryos had significantly higher indices $(10.2 \pm 1.0)$ than embryos derived in vivo $(6.5 \pm$ 0.8). Thus, the nuclear transfer procedure not only increases the incidence of apoptosis, but also disrupts its normal chronological pattern. Despite the fact that apoptosis is not evident until compaction in normal embryos developed in vivo, the blastomeres of the early precompaction cleavage stages possess the capacity for apoptosis, as the process can be induced by incubation in staurosporine (Weil et al., 1996; Matwee et al., 2000). The embryo 

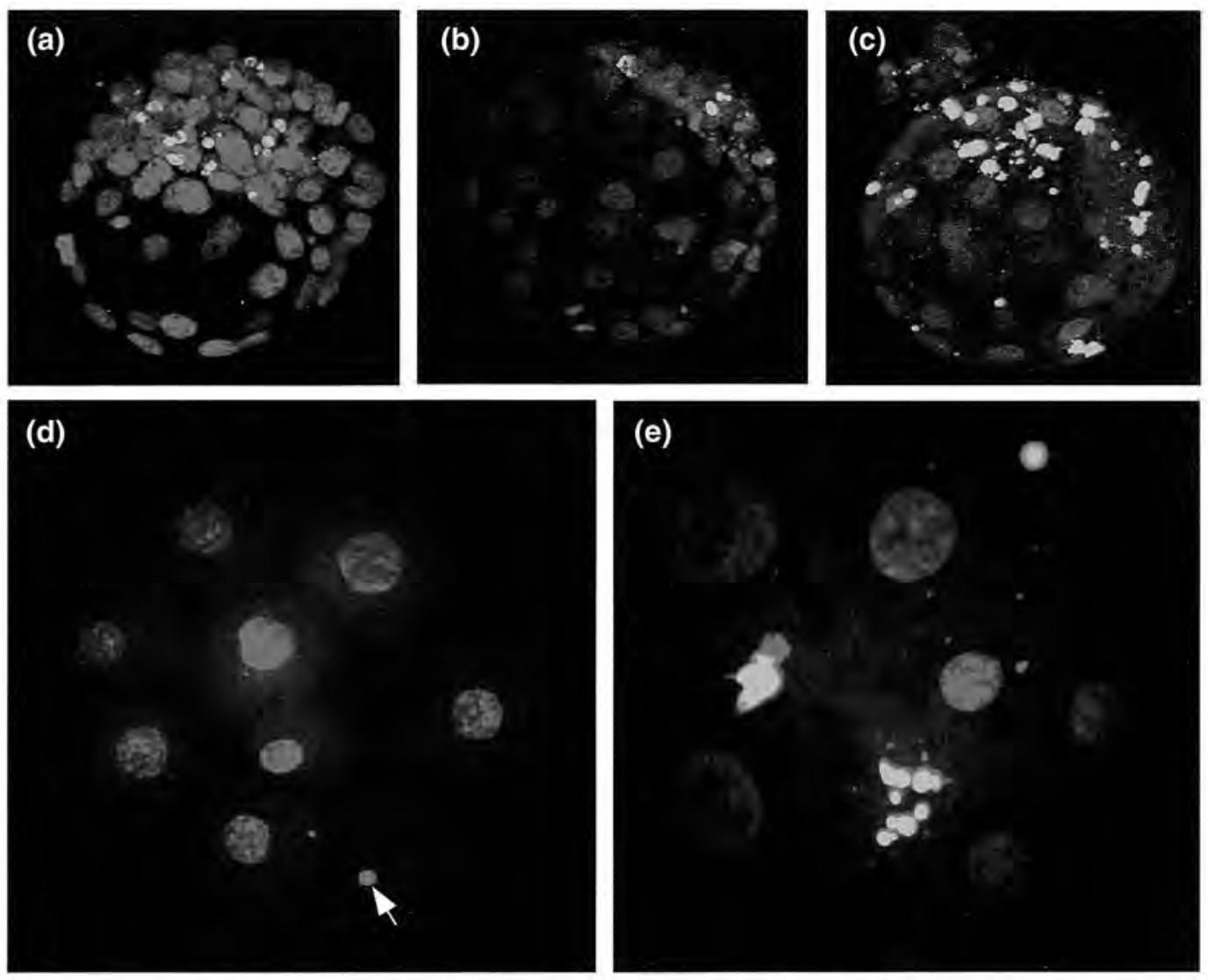

Fig. 3. Confocal laser scanning images of bovine embryos in which DNA has been stained with propidium iodide (red) and DNA fragmentation has been localized with TdT-mediated dUTP nick-end labelling (TUNEL) staining using fluorescein isothiocyanate (FITC) (green) labelled nucleotides. The embryos are (a) in vivo developed, (b) in vitro produced and (c) somatic nuclear transfer blastocysts and (d) in vivo developed and (e) somatic nuclear transfer 8-16-cell stages. Note the sporadic occurrence of apoptotic cells (nuclear condensation combined with positive TUNEL staining) in particular in the inner cell mass of the blastocysts. In addition, note the occurrence of apoptotic cells in the nuclear transfer cleavage stage embryo as opposed to cleavage stage embryo developed in vivo in which only a condensed nucleus without TUNEL staining is seen (arrow).

stage-specific onset of the normal apoptotic response in vivo may be due to an inadequate expression or activation of certain parts of the apoptotic machinery, or to extensive repression thereof. The accelerated onset of the different apoptotic markers observed in the nuclear transfer embryos indicates that the capacity for apoptosis is partly determined by the (donor) nucleus.

Several roles for apoptosis in preimplantation development have been proposed, mainly concerning elimination of abnormal or unwanted cells (Hardy, 1999; Betts and King, 2001). The observation of a decrease in the number of cells in combination with an increased incidence of apoptosis in the ICM of blastocysts produced in vitro indicates that cells are eliminated to a higher degree from this embryonic compartment in particular. This finding may reflect an increase of subcellular abnormalities in this important embryonic compartment provoked by handling in vitro. 


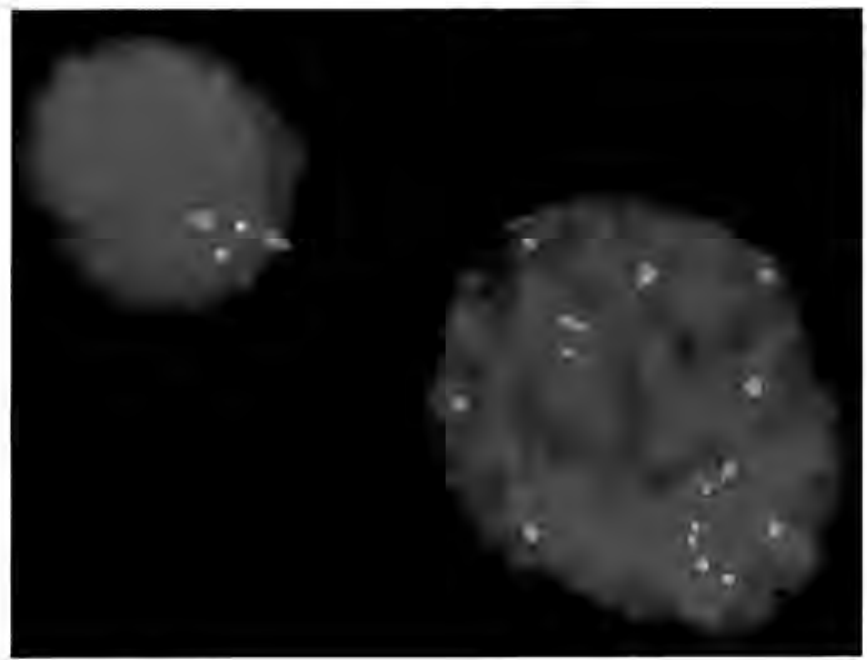

Fig. 4. Epifluorescence images of single nuclei from bovine blastocysts produced in vitro that are labelled by fluorescence in situ hybridization (FISH) with chromosome specific probes hybridizing to chromosomes 6 (green) and 7 (red). A normal diploid nucleus with two signals from each probe (upper left) and an octaploid nucleus with eight signals from each prope (lower right) are displayed.

\section{Chromosome abnormalities}

Embryos consisting only of polyploid or haploid cells are always eliminated in mammals, but karyotyping has clearly documented that single polyploid or haploid cells in otherwise normal diploid embryos are a frequent finding as early as the two-cell stage (for a review, see King, 1990). This finding may not be surprising as polyploid cells are also found normally in many organs of the mammalian body, most prominently in the liver. However, little is known about their biological significance or their lifespan in the adult, the fetus and, in particular, the embryo. Data from experimentally produced diploid-tetraploid mouse embryos indicate that polyploid cells at the morula and blastocyst stage are selected against in the embryo proper resulting in a preferential allocation to the trophoblast (Everett and West, 1998). These data taken together with reports of an inverse relationship between embryo quality and chromosome aberrations (King et al., 1987; Rubes et al., 1988) indicate that polyploid cells are a developmental disadvantage which under normal conditions may be regulated by segregation of aberrant cells to the trophoblast. However, these reports were produced using traditional karyotyping, which per se underestimates the frequency of mixoploidy. Therefore, the goal was to generate a more reliable estimate for the normal frequency of mixoploidy and polyploidy in embryos developed in vivo, using FISH with two chromosome-specific DNA probes (Fig. 4). The major advantage of this approach compared with karyotyping is that most, if not all, cells of an embryo can be evaluated for errors of ploidy.

The first estimates of chromosome abnormalities in bovine embryos developed in vivo generated by FISH analysis revealed that $25 \%$ of blastocysts collected on day 7 or day 8 after ovulation were mixoploid, that is, contained a large population of normal diploid cells and a small, in general $<10 \%$, population of haploid or polyploid cells (Viuff et al., 1999). This study included only 28 embryos, but it formed a starting point for further studies that were designed to elucidate at what stage of development the polyploid cells 
were formed and what their fate was later in development. The results from our analysis of 256 bovine embryos developed in vivo isolated on days 2, 3, 4 and 5 after ovulation revealed an increase in the frequency of mixoploidy (Viuff et al, 2001). Thus, the percentages of mixoploid embryos on days 2, 3, 4 and 5 were $5,13,16$ and $31 \%$, respectively. The corresponding percentages of polyploidy, that is, embryos in which all cells were polyploid, were $2,2,0$ and $0 \%$, which were significantly lower. Another important finding was that about $90 \%$ of 40 mixoploid embryos contained $<10 \%$ aberrant cells. Diploidy-triploidy was the most frequent mixoploid finding (50\%), whereas diploidy-haploidy, diploidy-tetraploidy, and diploidy-triploidy-haploidy plus diploidy-triploidy-tetraploidy were observed in 12.5, 25.0 and $12.5 \%$ of the embryos, respectively. The polyploid embryos of this study, of which only three were found, were triploid.

The first FISH data on chromosome aberrations in bovine embryos produced in vitro, which included 151 blastocysts isolated at days 7-8 after insemination in vitro, showed that $72 \%$ were mixoploid (Viuff et al., 1999). This was significantly more than in embryos derived in vivo. However, again, the frequency of aberrant cells was low in the mixoploid embryos: $83 \%$ contained $<10 \%$ aberrant cells, $13 \%$ contained $11-25 \%$, and only $4 \%$ contained $>25 \%$ aberrant cells. In a larger study of earlier in vitro produced developmental stages it was possible to document an increased rate of mixoploidy at about day 5 (Viuff et al., 2000): of 426 embryos, $22 \%$ were mixoploid at day $2,15 \%$ at day $3,16 \%$ at day 4 and $42 \%$ at day 5 . The corresponding percentages of polyploidy were $5,13,3$ and $0 \%$, which was markedly more than that seen in embryos developed in vivo. In 99 mixoploid embryos, diploidy-triploidy was the most frequent abnormality $(65 \%)$, whereas diploidy-tetraploidy and diploidy-triploidy-tetraploidy were observed in 11 and $24 \%$ of embryos, respectively. Among the polyploid embryos, triploidy was again the most common aberration. In addition to the lack of polyploid embryos at day 5, it was striking to observe that the development of polyploid embryos was significantly $(P<0.02)$ slower than that of the diploid and the mixoploid embryos. In addition, few, if any, polyploid embryos progressed beyond the third cell cycle, whereas the mixoploid embryos seemed to continue development unaffected beyond this stage (Viuff et al., 2001).

In addition, it was demonstrated that there was a significantly lower proportion of polyploid cells in the embryonic disc in comparison with the trophoblast of bovine embryos on both days $7-8$ and day 12 after insemination and that the level of polyploidy on days $7-8$ was significantly higher than on day 12 (Viuff et al., 2002). Thus, the polyploid cells may be confined to the trophoblast and make little contribution to the embryo itself. However, their functional significance is still unclear.

Thus, there are significant differences in the frequencies of polyploidy and mixoploidy between embryos developed in vivo and embryos produced in vitro. Therefore, the aim of the following study was to use the occurrence of chromosome abnormalities as a marker for evaluating the effect of performing oocyte maturation versus embryo culture in vitro. Oocytes were either matured in vivo in superovulated follicles and aspirated under ultrasound guidance or matured in vitro, and both categories of oocyte were processed for fertilization and culture in vitro (Dielemann et al., 2002). On the basis of the results from embryos developed in vivo (Viuff et al., 1999), embryos with $<5 \%$ aberrant cells were classified as normal. The preliminary results indicate that significantly more of the blastocysts produced from oocytes matured in vivo were normal in comparison with blastocysts produced from oocytes matured in vitro. Furthermore, blastocysts from oocytes matured in vitro contained fewer cells than blastocysts from oocytes developed in vivo (54 versus 96 cells per normal blastocyst). Correspondingly, it has been demonstrated that oocytes matured in vivo produce significantly more blastocysts than oocytes matured in vitro after in vitro fertilization and 
subsequent culture (Rizos et al., 2002). Interestingly, the same investigation showed that in vitro culture of zygotes fertilized in vivo results in the same blastocyst rate as in vitro culture of zygotes produced in vitro. However, the blastocysts originating from zygotes developed in vivo had a higher capacity to tolerate vitrification. Together, these data indicate that maturation of oocytes in vitro certainly does affect the development of the embryo to the blastocyst stage with respect to both rate and quality.

Finally, the occurrence of chromosome abnormalities in bovine embryos reconstructed by nuclear transfer from embryonic cells was determined (Booth et al., 2000). The proportion of completely normal embryos was the same as among embryos produced in vitro, but among embryos with chromosome aberrations, the nuclear transfer embryos were more severely affected. These abnormalities may be involved in the marked embryonic loss inherent to nuclear transfer.

\section{Embryonic development after hatching}

From the data presented here it is clear that a blastocyst, despite having an excellent appearance under the stereomicroscope, may have undetected abnormalities that have an impact on its developmental competence. Therefore, the relevance of using rates of expanded or even hatched blastocysts as end points of evaluation can be questioned. It might be advantageous if further characteristics of embryonic development after hatching in vitro could be added to the list of parameters used for assessment of embryonic competence.

The basis for such long-term evaluation in vitro of bovine embryos was created by first defining a set of milestones of bovine embryonic development in vivo up to day 21 after ovulation and, second, evaluating the capacity of bovine embryos produced in vitro to complete these milestones under long-term in vitro culture (Vajta et al., 2000, 2001; Hyttel et al., 2002). Bovine embryos developed in vivo were collected after slaughter on day 14 and day 21 after ovulation and processed for TEM or immunohistochemistry for germ layer characterization (cytokeratin 8 for potential ectoderm, alpha fetoprotein for potential endoderm and vimentin for potential mesoderm). On day 8 after insemination, embryos produced in vitro were transferred to culture on reconstituted collagen matrix in SOFaaci (Holm et al, 1999) supplemented with $50 \%$ FCS at $40 \% \mathrm{O}_{2}, 5 \% \mathrm{CO}_{2}$ and $55 \% \mathrm{~N}_{2}$ and processed for TEM at days 8-29 after insemination.

Embryos developing in vivo on day 14 were ovoid to filamentous and ranged from about 0.5 to $19.0 \mathrm{~mm}$ in total length (Fig. 5). The embryos displayed a complete hypoblast lining of the trophoblast, and an epiblast was inserted into the trophoblast with tight junctions present between adjacent epiblast cells, and between peripheral epiblast and trophoblast cells. In some embryos, the epiblast was more or less covered by foldings of trophoblast in the process of forming the amniotic cavity. Cytokeratin 8 was localized to the trophoblast and the hypoblast underlying the epiblast, alpha fetoprotein to some, but not all, cells of the hypoblast underlying the trophoblast, and vimentin to some, but not all, cells of the epiblast. On day 21 , the length of the embryo proper, not the full conceptus, ranged from about 0.6 to $6.0 \mathrm{~mm}$. The developmental stages ranged from embryos presenting a primitive streak and formation of the neural grove to those presenting a neural tube, and up to 14 somites and allantois development. These embryos showed the gradual formation of endoderm, mesoderm and ectoderm as well as differentiation of paraxial, intermediate and latetal plate mesoderm and somites. Cytokeratin 8 was localized to the trophectoderm, the hypoblast and the surface and neural ectoderm, and alpha fetoprotein to the hypoblast, but not the final endoderm, the intensity increasing with development. Vimentin was initially localized to some, but not all, cells positioned particularly in the ventral region of the primitive streak, 

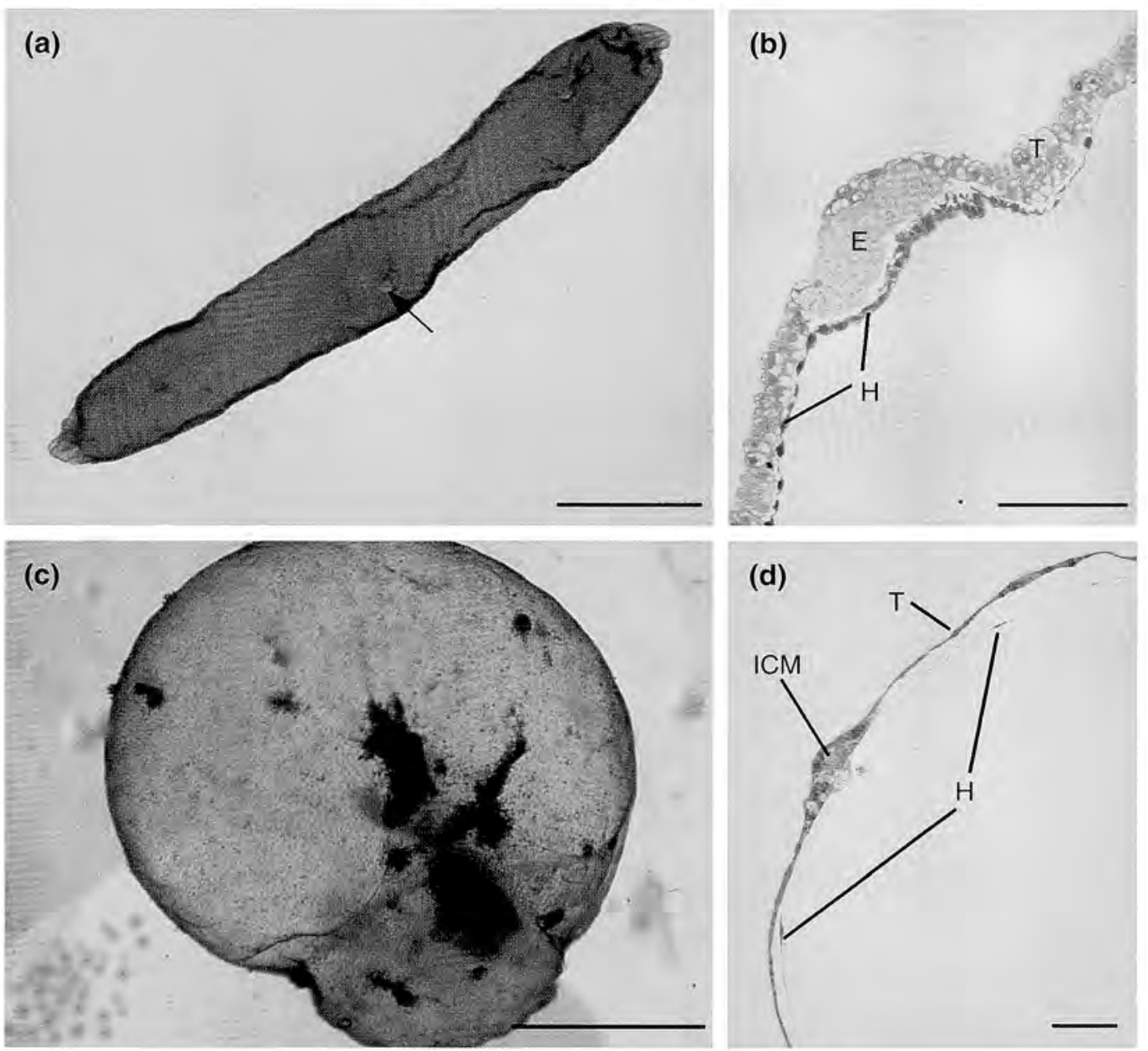

Fig. 5. Stereomicroscope image of (a) a bovine conceptus developed in vivo on day 14 after fertilization. Semi-thin Epon section from (b) the region of the embryonic disc of the same embryo displaying trophoblast $(\mathrm{T})$, epiblast $(\mathrm{E})$ and hypoblast $(\mathrm{H})$. Stereomicroscope image of $(\mathrm{c})$ an in vitro produced and in vitro grown bovine conceptus on day 23 after fertilization. Note the lack of elongation. Semi-thin Epon section of (d) another bovine conceptus grown in vitro on day 11 after fertilization displaying trophoblast $(\mathrm{T})$, inner cell mass (ICM) and development of hypoblast $(\mathrm{H})$ from the ICM. Scale bars represent $(\mathrm{a}, \mathrm{c}) 1.0 \mathrm{~mm}$ and $(\mathrm{b}, \mathrm{d}) 0.1 \mathrm{~mm}$.

to presumptive final endoderm cells inserted into the upper portion of the hypoblast, and to mesoderm.

Bovine embryos developing in vitro remained spherical, and up to day 11 after insemination, half of the embryos displayed hypoblast formation (Fig. 5) and about one third presented abortive establishment of an epiblast by penetration of cells from the ICM mass through the trophoblast. However, in all cases, this process was associated with degeneration of the ICM, and from day 12, the ICM had degenerated in all embryos. From day 14, a 'foot' process gradually developed as a trophoblast outgrowth on the collagen matrix. A compact cell mass, from which several vesicles were formed, was established at the site of the 'foot' process. 
The data demonstrate that initial bovine embryonic development after hatching includes well-defined milestones of which only hypoblast formation can be achieved by the present conditions for long-term culture of embryos in vitro.

\section{Conclusion}

A variety of techniques allow for detection of subcellular deviations, such as aberrant gene activation and increased occurrence of apoptosis and chromosome abnormalities, in biotechnologically manufactured embryos arising from, for example, in vitro production or reconstruction by nuclear transfer. With the rapid expansion of the area of bioimaging in which techniques from morphology and molecular biology combine, the coming years will offer a range of exciting possibilities including the potential observation of subcellular function of live embryos by means of multiphoton confocal microscopy.

The work was supported by the Danish Agricultural and veterinary research Council and A. B. Fond. The authors are grateful to I. Nielsen for excellent support for preparation and image processing.

\section{References}

Baran V, Flechon JE and Pivko J (1996) Nucleologenesis in the cleaving bovine embryo: immunocytochemical aspects Molecular Reproduction and Development 44 63-70

Baran V, Vignon X, LeBourhis D, Renard IP and Fléchon JE (2002) Nucleolar changes in bovine nucleotransferred embryos Biology of Reproduction $\mathbf{6 6}$ 534-543

Betts DH and King WA (2001) Genetic regulation of embryo death and senescence Theriogenology 55 171-191

Booth BJ, Viuff D, Thomsen PD, Holm P, Greve T and Callesen H (2000) Ploidy of bovine nuclear transfer blastocysts reconstructed using in vitro produced blastomere donors Cloning 2 63-68

Brison DR and Schultz RM (1997) Apoptosis during mouse blastocyst formation: evidence for a role for survival factors including transforming growth factor alpha Biology of Reproduction 56 1088-1096

Byrne AT, Southgate J, Brison DR and Leese HI (1999) Analysis of apoptosis in the preimplantation bovine embryo using TUNEL Journal of Reproduction and Fertility 117 97-105

Camous S, Kopecny V and Flechon JE (1986) Autoradiographic detection of the earliest stage of $[3 \mathrm{H}]$-uridine incorporation into the cow embryo Biology of the Cell 58 195-200

Devreker F and Hardy K (1997) Effects of glutamine and taurine on preimplantation development and cleavage of mouse embryos in vitro. Biology of Reproduction $\mathbf{5 7} 921-928$

Dielemann SJ, Hendriksen PJM, Viuff D et al (2002) Effects of in vivo prematuration and in vivo final maturation on developmental capacity and quality of preimplantation embryos Theriogenology 57 5-20
Everett CA and West JD (1998) Evidence for selection against tetraploid cells in tetraploid/diploid mouse chimaeras before the late blastocyst stage Genetic Research 72 225-228

Fair T, Hyttel P and Greve T (1995) Bovine oocyte diameter in relation to maturational competence and transcriptional activity Molecular Reproduction and Development 42 437-442

Fair T, Hyttel P, Greve T and Boland M (1996) Nucleolus structure and transcriptional activity in relation to oocyle diameter in cattle Molecular Reproduction and Development 43 503-512

Gavrieli Y, Sherman Y and Ben Sasson SA (1992) Identification of programmed cell death in situ via specific labeling of nuclear DNA fragmentation Journal of Cell Biology 119 493-501

Gjørret JO, Avery B, Larsson L-I, Schellander K and Hyttel P (2001) Apoptosis in bovine blastocysts produced in vivo and in vitro. Theriogenology 55 321 (Abstract)

Gjørret JO, Wengle J, King WA, Schellander $\mathrm{K}$ and Hyttel P (2002) Occurrence of apoptosis in bovine embryos reconstructed by nuclear transfer or derived in vivo. Theriogenology 57495 (Abstract)

Grasl-Kraupp B, Ruttkay-Nedecky B, Koudelka H, Bukowska K, Bursch W and Schulte-Hermann R (1995) In situ detection of fragmented DNA (TUNEL assay) fails to discriminate among apoptosis, necrosis and autolytic cell death: a cautionary note Hepatology 21 1465-1468

Hardy K (1997) Cell death in the mammalian blastocyst Molecular Human Reproduction 3 919-925

Hardy K (1999) Apoptosis in the human embryo Reviews of Reproduction 4 125-134

Hardy K, Handyside AH and Winston RM (1989) The human blastocyst: cell number, death and allocation 
during late preimplantation development in vitro. Development 107 597-604

Hengartner MO (2000) The biochemistry of apoptosis Nature $407770-776$

Holm P, Booth PJ and Callesen H (1999) High bovine blastocyst development in a static in vitro production system using SOFaa medium supplemented with sodium citrate and myo-inositol with or without serum-proteins Theriogenology 52 683-700

Hozak P, Cook PR, Schofer C, Mosgoller W and Wachtler F (1994) Site of transcription of ribosomal RNA and intranucleolar structure in Hela cells. lournal of Cell Science 107 639-648

Hyttel P, Viuff D, Avery B and Greve T (1996) Transcription and cell cycle dependent development of intranuclear bodies and granules in 2-cell bovine embryos Journal of Reproduction and Fertility 108 $263-270$

Hyttel P, Laurincik J, Viuff D, Hendriksen PJM, Dieleman SJ, Besenfelder $\mathrm{U}$ and Schellander $\mathrm{K}$ (2001a) Nucleolar proteins and ultrastructure in bovine embryos in vivo. Theriogenology $\mathbf{5 5} 450$ (Abstract)

Hyttel P, Laurincik J, Zakhartchenko V, Stojkovic M, Wolf E, Müller M, Ochs RL and Brem G (2001b) Nucleolar protein allocation and ultrastructure in bovine embryos produced by nuclear transfer from embryonic cells Cloning 3 69-81

Hyttel P, Alexopoulos NI and Lewis 1 (2002) Posthatching development of bovine embryos in vivo and in vitro. Theriogenology 57497 (Abstract)

Jurisicova A, Varmuza S and Casper RF (1996) Programmed cell death and human embryo fragmentation Molecular Human Reproduction 2 93-98

King WA (1990) Chromosome abnormalities and pregnancy failure in domestic animals. In Advances in Veterinary Science and Comparative Medicine 34 229-250 Ed. RA McFeely. CA: Academic Press, San Diego

King WA, Guay P and Picard L (1987) A cytogenetical study of 7-day-old bovine embryos of poor morphological quality Genome 29 160-164

King WA, Niar A, Chartrain I, Betteridge KJ and Guay P (1988) Nucleolus organizer regions and nucleoli in preattachment bovine embryos Journal of Reproduction and Fertility $\mathbf{8} 87-95$

Kopecny V and Niemann H (1993) Formation of nuclear microarchitecture in the preimplantation bovine embryo at the onset of transcription: implications for biotechnology Theriogenology 39 109119

Kopecny V, Flechon JE, Camous S and Fulka J, Jr (1989) Nucleologenesis and the onset of transcription in the eight-cell bovine embryo: fine-structural autoradiographic study Molecular Reproduction and Develop. ment 1 79-90

Laurincik J, Thomsen PD, Hay-Schmidı A, Avery B, Greve T, Ochs RL and Hyttel P (2000) Nucleolar pro- teins and nuclear ultrastructure in pre-implantation bovine embryos produced in vitro. Biology of Reproduction 62 1024-1032

Laurincik J, Zakhartchenko V, Stojkovic M, Brem G, Wolf $E$, Müller M, Ochs RL and Maddox-Hyttel P (2002) Nucleolar protein allocation and ultrastructure in bovine embryos produced by nuclear transfer from granulosa cells Molecular Reproduction and Development 61 477-487

Leist M and Jaattela M (2001) Four deaths and a funeral: from caspases to alternative mechanisms Nature Reviews of Molecular and Cell Biology $2589-598$

Long CR, Dobrinsky JR, Garrett WM and Johnson LA (1998) Dual labeling of the cytoskeleton and DNA strand breaks in porcine embryos produced in vivo and in vitro. Molecular Reproduction and Development 51 59-65

McEvoy TG, Robinson J] and Sinclair KD (2001) Developmental consequence of embryo and cell manipulation in mice and farm animals Reproduction 122 507-518

Makarevich AV and Markkula M (2002) Apoptosis and cell proliferation potential of bovine embryos stimulated with insulin-like growth factor 1 during in vitro maturation and culture Biology of Reproduction 66 386-392

Matwee C, Betts DH and King WA (2000) Apoptosis in the early bovine embryo Zygote 8 57-68

Memili E and First NL (2000) Zygotic and embryonic gene expression in cow: a review of timing and mechanisms of early gene expression as compared with other species Zygote 8 87-96

Moley $\mathrm{KH}$, Chi MM, Knudson CM, Korsmeyer SJ and Mueckler MM (1998) Hyperglycemia induces apoptosis in pre-implantation embryos through cell death effector pathways Nature Medicine 4 1421-1424

Pampier S, Vanderheyden I, McCracken JE, Vesela J and De HR (1997) Increased cell death in rat blastocysts exposed to maternal diabetes in utero and to high glucose or fumor necrosis factor-alpha in vitro. De velopment $1244827-4836$

Plante L, Plante C, Shepherd DL and King WA (1994) Cleavage and ${ }^{3} \mathrm{H}$-uridine incorporation in bovine embryos of high in vitro developmental potential Molecular Reproduction and Development 39375 383

Rizos D, Ward F, Duffy P, Boland $\mathrm{M}$ and Lonergan $\mathrm{P}$ (2002) Consequences of bovine oocyte maturation, ferilization or early embryonic development in vitro versus in vivo: implications for blastocyst yield and blastocyst quality Molecular Reproduction and Development 61 234-248

Rubes J, Zák M, Horinová Z and Machatková M (1988) Cytogenetic examination of bovine embryos of different quality. Bth European Colloquium on Cytogenetics of Domestic Animals 134-138

Schultz RM (1993) Regulation of zygotic gene activation in the mouse Bioessays 15 531-538 
Vajta G, Hyttel P and Trounson A (2000) Post-hatching development of in vitro produced bovine embryos on agar and collagen gels Animal Reproduction and Science 208 60-61

Vajta G, Alexopoulos NI, Hall VJ, Lewis IM, French AJ, Denham MS and Trounson AO (2001) In vitro development of IVM/IVF bovine embryos cultured beyond 30 days in different protein sources Theriogenology 55344 (Abstract)

Viuff D, Avery B, Greve T, King WA and Hyttel P (1996) Transcriptional activity in in vitro produced bovine 2- and 4-cell embryos Molecular Reproduction and Development 43 171-179

Viuff D, Hyttel P, Avery B, Vajta G, Greve T, Callesen H and Thomsen PD (1998) Ribosomal ribonucleic acid is transcribed at the 4-cell stage in in vitro produced bovine embryos Biology of Reproduction $\mathbf{5 9}$ 626-631

Viuff D, Rickords L, Offenberg H, Hyttel P, Avery B, Greve T, Olsaker I, Williams IL, Callesen $H$ and Thomsen PD (1999) A high proportion of bovine blastocysts produced in vitro are mixoploid Biology of Reproduction $601273-1278$

Viuff D, Greve T, Avery B, Hyttel P, Brockhoff PB and Thomsen PD (2000) Chromosome aberrations in in vitro-produced bovine embryos at days 2-5 post-insemination Biology of Reproduction $631143-$ 1148

Viuff D, Hendriksen PJM, Vos PLAM, Dieleman SJ, Bibby BM, Greve T, Hyttel P and Thomsen PD (2001) Chromosomal abnormalities and developmental kinetics in in vivo-developed cattle embryos at days 2-5 after ovulation Biology of Reproduction 65 204-208

Viuff D, Palsgaard A, Rickords L, Lawson LG, Greve T, Schmidt M, Avery B, Hyttel P and Thomsen PD (2002) Bovine embryos contain higher proportion of polyploid cells in the trophectoderm than in the embryonic disc Molecular Reproduction and Development 62 483-488

Wachtler F and Stahl A (1993) The nucleolus: a structural and functional interpretation Micron 24 473-505

Weil M, Jacobson MD, Coles HS, Davies TJ, Gardner RL, Raff KD and Raff MC (1996) Constitutive expression of the machinery for programmed cell death Journal of Cell Biology 133 1053-1059

Wyllie AH, Kerr JF and Currie AR (1980) Cell death: the significance of apoptosis Internalional Review of Cytology 68 251-306 